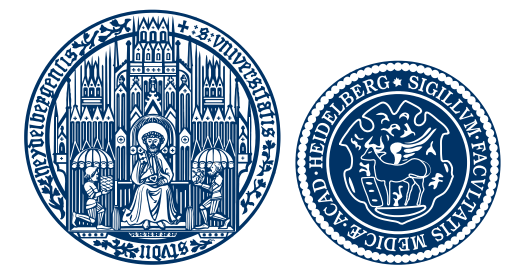

UniversitätsKlinikum Heidelberg

Randomisierte kontrollierte Therapiestudie zur psychodynamischen und kognitiv-verhaltenstherapeutischen Behandlung von weiblichen Jugendlichen und jungen Erwachsenen mit Bulimia nervosa und atypischer Bulimia nervosa



\title{
Elternaufklärung
}

Hauptverantwortlicher Projektleiter

Dr. med. Dipl.-Psych. K.-T. Kronmüller

Psychiatrische Klinik der Universität Heidelberg

Voßstr. 4

69115 Heidelberg

Tel. (06221) 565938 
Sehr geehrte Eltern,

Ihre Tochter hat die Gelegenheit, an einer Studie zur Psychotherapie der Bulimie und atypischen Bulimie bei Jugendlichen und jungen Erwachsenen teilzunehmen. Ziel der Untersuchung ist es, die Wirkweisen von zwei Formen von Psychotherapie miteinander zu vergleichen. Außerdem wollen wir herausfinden, welchen Patientinnen welche Therapie am besten hilft. Ziel ist es, allen Patientinnen mit Bulimie eine für sie wirkungsvolle Therapie anbieten zu können.

Die Studie wird von Psychiatrie des Universitätsklinikums Heidelberg gemeinsam mit dem Institut für Analytische Kinder- und Jugendlichen-Psychotherapie Heidelberg und der Abteilung für Psychosomatik und Psychotherapie der Universitätskliniken Göttingen durchgeführt.

Wenn Sie der Teilnahme zustimmen, würden wir Ihre Tochter ausführlich zu ihren Beschwerden und ihrer Lebenssituation befragen und sie um die Beantwortung einiger Fragebögen zu diesem Thema bitten.

Anschließend würde Ihre Tochter nach dem Zufallsprinzip einer von zwei Arten von Psychotherapie zugewiesen. Dies ist aus forschungstechnischen Gründen notwendig, da nur auf diese Weise die spezifische Wirksamkeit der beiden Therapieverfahren miteinander verglichen werden kann. Beide Therapien dauern 60 Stunden, gehen über ein Jahr und finden jeweils wöchentlich eine bis zwei Stunden an einem mit Ihrer Tochter (und eventuell mit Ihnen) abzusprechenden Termin statt. Beide Therapien haben sich bei Erwachsenen als wirksam erwiesen, unterscheiden sich aber untereinander. Wir können vor der Behandlung nicht sagen, ob eine der beiden Therapien für Ihre Tochter die bessere ist.

Im Laufe der Therapie werden wir von Ihrer Tochter nochmals persönlich bzw. durch die Beantwortung einiger Fragebögen Auskunft über deren aktuellen Beschwerden einholen. Zudem werden einige der Therapiesitzungen auf Video aufgezeichnet und ausgewertet. Dies dient der Kontrolle der Therapeuten und der Optimierung der Therapie.

Zum Abschluss der Behandlung sowie 6 Monate und ein Jahr nach deren Beendigung werden wir einen erneuten Termin mit Ihrer Tochter vereinbaren, um sie wiederum ausführlich zu ihren Beschwerden und ihrer Lebenssituation zu befragen, und sie um die Beantwortung einiger Fragebögen zu diesen Themen bitten.

Zudem möchten wir, das Einverständnis Ihrer Tochter vorausgesetzt, am Anfang, nach 30 Therapiestunden und am Ende der Therapie sowie 6 Monate und 1 Jahr nach Beendigung Ihre Einschätzung zu deren Beschwerden und der Familiensituation mittels Fragebögen erfragen, um zusätzlich relevante Informationen zu erhalten. 
Die erhobenen Informationen werden mittels standardisierter Erfassungsbögen dokumentiert. Die Daten aller beteiligten Patientinnen und Eltern werden an der Psychiatrischen Klinik der Universität Heidelberg pseudonymisiert ausgewertet. Die Vorschriften über die ärztliche Schweigepflicht und den Datenschutz werden im Rahmen der Studie selbstverständlich eingehalten.

Die Teilnahme an dieser Untersuchung erfolgt freiwillig. Ihre Tochter und Sie haben zu jedem Zeitpunkt das Recht, die Antwort auf die gestellten Fragen zu verweigern oder Ihr Einverständnis für die Durchführung der Untersuchung bzw. für die Teilnahme an der Studie insgesamt zu widerrufen. Die Nichtteilnahme führt zu keinen Nachteilen für die weitere psychotherapeutische Versorgung Ihrer Tochter. Bei Rücktritt wird bereits gewonnenes Material vernichtet, es sei denn, Sie und Ihre Tochter stimmen zu, dass Sie trotz Ihres Rücktritts mit dessen Auswertung einverstanden sind.

Die Durchführung der Aufklärung sowie die Einwilligung in die Teilnahme an der Studie werden durch Ihre Unterschrift, die der Patientin sowie des aufklärenden Studienmitarbeiters belegt.

Durch die Behandlung entsteht kein zusätzliches Risiko. Es besteht vielmehr die Chance, durch die Therapie die Beschwerden Ihrer Tochter zu verringern oder zu beseitigen.

Für Rückfragen stehen wir Ihnen persönlich oder telefonisch gern zur Verfügung.

Mit freundlichen Grüßen

Dr. med. Dipl-Psych. Klaus Kronmüller

Psychiatrie

Universitätsklinikum Heidelberg

Vossstraße 4

69115 Heidelberg 


\section{EINWILLIGUNGSERKLÄRUNG - ELTERN}

Randomisierte kontrollierte Therapiestudie zur psychodynamischen und kognitivverhaltenstherapeutischen Behandlung von weiblichen Jugendlichen und jungen Erwachsenen mit Bulimia nervosa und atypischer Bulimia nervosa

Patientin:

Name

Vorname

Geburtsdatum

Mutter:

Name

Vorname

Geburtsdatum

Anschrift/ Telefon

Vater:

Name

Vorname

Geburtsdatum

Anschrift/ Telefon (falls abweichend von Mutter) 
Hiermit erkläre ich mein Einverständnis zur Teilnahme meiner Tochter an der oben genannten klinischen Studie zur Psychotherapie der Bulimie.

Ich erkläre ebenfalls mein Einverständnis damit, im Rahmen der Studie befragt zu werden. Ich wurde über den Inhalt der Studie und die geplanten Maßnahmen informiert und hatte Gelegenheit, den mir ausgehändigten Aufklärungsbogen sorgfältig zu lesen. Die Studienteilnahme meiner Tochter erfolgt freiwillig, alle meine Fragen im Zusammenhang mit der Studie wurden hinreichend beantwortet. Mir ist bekannt, dass ich meine Einwilligung jederzeit ohne Angaben von Gründen widerrufen kann, ohne dass mir oder meiner Tochter hieraus irgendwelche Nachteile entstehen.

Ich wurde darüber aufgeklärt und stimme zu, dass meine und die meiner Tochter in der Studie erhobenen Daten in pseudonymisierter Form aufgezeichnet (und ggf. weitergegeben) werden können. Dritte erhalten jedoch keinen Einblick in Originalunterlagen. Bei der Veröffentlichung von Ergebnissen der Studie wird der Name meiner Tochter und meine Name ebenfalls nicht genannt.

Heidelberg, den

(Unterschriften der Eltern)

Heidelberg, den 